\title{
Withdrawal and drainage of thin film flow on a vertical cylinder
}

\author{
A. M. Siddiqui ${ }^{1}$, M. Akram ${ }^{2}$, K. N. Memon ${ }^{2 \star}$, S. Islam ${ }^{3}$ and Khalid khan ${ }^{4}$ \\ ${ }^{1}$ Pennsylvania State University, York Campus, Edgecombe 17403, USA. \\ ${ }^{2}$ Mathematics, COMSATS Institute of Information Technology, Islamabad, Pakistan. \\ ${ }^{3}$ Department of Mathematics, Abdul Wali Khan University, Mardan, Pakistan. \\ ${ }^{4}$ Department of Science and Information Technology Govt; KPK, Peshawar, Pakistan.
}

Accepted 17 August, 2012

\begin{abstract}
The thin film flow of a power law fluid on a vertical cylinder for a lift and a drainage problem is studied. The goverming nonlinear differential equations have been derived from the continuity, momentum and constitutive equations. The resulting equations are then solved using binomial series method. Series solutions have been obtained for velocity, volume flow rate and average velocity in both cases. The graphical results for velocity profile is discussed and examined for different parameters of interest.
\end{abstract}

Key words: Thin film flow, power law fluid, binomial series method.

\section{INTRODUCTION}

Over the years, non-Newtonian fluids have been considered due to their practical importance in industry, medicine and sciences. Few examples of such fluids are: tooth paste, paints, greases, blood, drilling mud, clay coatings, polymer melts etc. No single model can exhibit all the properties of fluids, for this purpose different differential and integral constitutive equations have been proposed (Deshpande and Barigou, 2001; Kemiha et al., 2006; Jie and Xi-Yun, 2006; Mahomed et al., 2007). Among these, the power law model has attained much attention of the researchers because of its wide spread industrial applications (Yong-Li et al., 2009; Kapur, 1963; Nejat et al., 2011; Ghoreishy and Razavi, 1998).

Study of thin film flow has received significant attention due to practical concentration of these flow's in physical and biological sciences vaccinated by thin film flows. Many researchers have grappled with the analyze these type of flows since their formulation. The nonNewtonian fluids have been used by researchers (Siddiqui et al., 2006a,b; He, 2008; Hayat and Sajid, 2007, 2008; Siddiqui et al., 2007) for thin film flow to investigate and solve them analytically and numerically.

Most of the occurring natural and industrial problems

\footnotetext{
*Corresponding author. E-mail: kamrannazir025@yahoo.com.
}

when modeled show nonlinearity and few of them show linearity. Non-linearity increases the mathematical complexity of the problems which reduces the chance of getting exact solutions. In view of such difficulties, in past three decades, researchers and scientists developed numerous analytic and numerical techniques to overcome non-linearity and get approximate solutions. Various analytical techniques such as binomial series method, perturbation method (PM), Adomian decomposition method (ADM), variational iteration method (VIM), homotopy analysis method (HAM), optimal homotopy analysis method (OHAM), homotopy perturbation method (HPM), optimal homotopy perturbation method (OHPM) and some others methods have proven to be valuable tools to understand the complexity of these type of problems (Siddiqui, 2006a, b; $\mathrm{He}, 2008,2011,2012$; Siddiqui et al., 2007; Dita and Grama, 1997). These techniques have found plentiful applications in industry and technology. In this paper, we shall use binomial series method (Coolidge, 1949) to analyze the flow behavior during withdrawal and drainage of power law fluid on a vertical cylinder.

In this paper, we investigate the thin film flow for lift and drainage on a vertical cylinder, two cases are discussed Newtonian and power law fluid, respectively. In Newtonian case, we find the exact solution while in power law series solution is obtained. According to the best of 
our knowledge, the solution of the problem has not been reported in the literature.

This study is organized as follows. Subsequently, the study presents the governing equation of the fluid model. Then, the problem under consideration is formulated and solution for the lifting case is given. Thereafter, it reserved for the solution of the drainage case. Afterwards, deals with results and discussion. Finally concluding remarks are given.

\section{BASIC EQUATIONS}

The basic equations, governing the flow of incompressible power law fluid neglecting the thermal effects, are:

$\nabla \cdot \mathbf{V}=0$

$\rho \frac{D \mathbf{V}}{D t}=\rho \mathbf{f}-\nabla p+\operatorname{div} \mathbf{S}$,

where $\rho$ is the constant density, $\mathbf{V}$ is the velocity vector, $\mathbf{f}$ is the body force, $p$ is the pressure, $\mathbf{S}$ is the extra stress tensor and $\frac{D}{D t}$ is denoting the material time derivative derivative. As discussed in Bird et al. (1987), the stress tensor defining a power law fluid is given by:

$$
\begin{aligned}
& S=\mu_{\text {eff }} A_{1}, \\
& \mu_{\text {eff }}=\eta \mid \sqrt{\left.\frac{\operatorname{tr}\left(A_{1}^{2}\right)}{2}\right|^{n-1},}
\end{aligned}
$$

and where $\eta$ is the coefficient of viscosity and $n$ is the Power law index. The Rivilin-Ericksen tensor, $\mathbf{A}_{\mathbf{1}}$ is defined by:

$$
\mathbf{A}_{1}=(\nabla \mathbf{V})+(\nabla \mathbf{V})^{T}
$$

Remark: On behalf of consequent model for $n<1$, the fluid is "pseudoplastic" for model or "shear thinning" for $n>1$ the fluid is "dilatant" or "shear-thickening" and for $n=1$ the Newtonian fluid is recovered.

\section{FORMULATION OF THE PROBLEM AND SOLUTION FOR LIFTING CASE}

Consider a container filled with power law fluid. A wide cylinder moves vertically upward through container with constant velocity $U_{0}$. Since the cylinder moves upward, it picks up a thin fluid film of

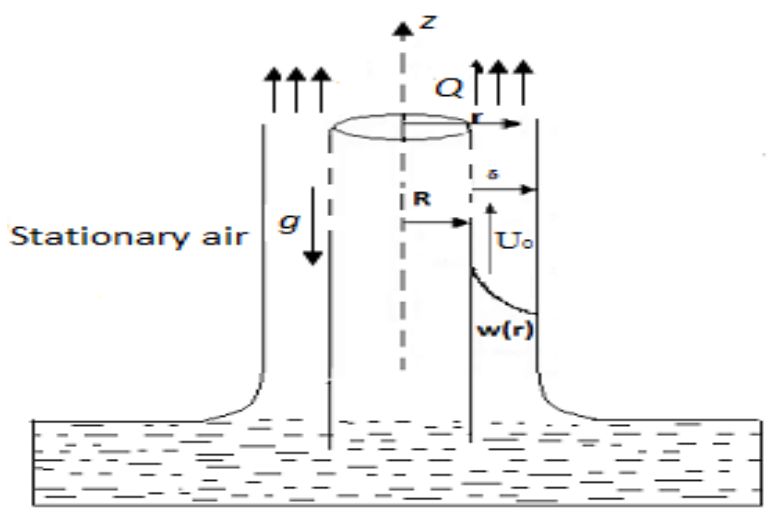

Figure 1. Geometry of the flow of moving cylinder through a power law fluid.

thickness $\delta$. Due to gravity, the fluid film tends to drain down the cylinder. We choose an $r z$-coordinate system such that $r-$ axis is normal to the cylinder axis and $z$-axis along the cylinder axis in upward direction as shown in Figure 1. Assuming that the flow is steady, laminar and uniform; and surface tension effects are negligible, the only velocity component is in $z$ - direction. Accordingly we assume that,

$\mathbf{V}=[0,0, w(r)], \quad \mathbf{S}=S(r)$.

Using Equation (6), the continuity Equation (1) is identically satisfied and the momentum Equation (2) reduce to,

$r$-component: $0=-\frac{\partial p}{\partial r}$

$\theta$-component: $0=-\frac{\partial p}{\partial \theta}$

$z$-component: $\frac{\partial p}{\partial z}=\frac{\eta}{r} \frac{\partial}{\partial r}\left(r\left|\frac{\partial w}{\partial r}\right|^{n-1} \frac{\partial w}{\partial r}\right)-\rho g$.

Equations (7) and (8) imply that $p=p(z)$ only. Assume that pressure the $p$ is atmospheric pressure, that is, $p$ is zero (gauge pressure) everywhere. As we are discussing the flow problem, we take $\frac{\partial w}{\partial r}$ positive. Thus Equation (9) reduces to,

$0=\frac{\eta}{r} \frac{d}{d r}\left(r\left(\frac{d w}{d r}\right)^{n}\right)-\rho g$,

which is non linear differential equation. The associated boundary conditions are:

$\frac{d w}{d r}=0 \quad$ at $\quad r=R+\delta$, 
$w=U_{0} \quad$ at $\quad r=R$

Introducing dimensionless parameters,

$r^{*}=\frac{r}{R}, \quad w^{*}=\frac{w}{U_{0}}$,

By using Equation (13) in Equation (10) and boundary conditions (11) and (12), we achieve after dropping the superscript '* ',

$\frac{1}{r} \frac{d}{d r}\left(r\left(\frac{d w}{d r}\right)^{n}\right)=S_{t}$,

$\frac{d w}{d r}=0 \quad$ at $\quad r=M$,

$w=1 \quad$ at $\quad r=1$,

where $S_{t}=\frac{\rho g R^{2}}{\mu_{e f f} U_{0}}$ is the Stokes' number, $\mu_{e f f}=\frac{\eta}{\left(R / U_{0}\right)^{n-1}}$

is power law fluid parameter and $M=1+\frac{\delta}{R}$. Integrating

Equation (14) with respect to $r$ and using boundary condition (15), we obtain,

$\frac{d w}{d r}=\left(\frac{S_{t}}{2}\right)^{\frac{1}{n}}\left(r-\frac{M^{2}}{r}\right)^{\frac{1}{n}}$.

The sign of $\frac{d w}{d r}$ is always opposite to that of $r$ because as $r$ increases, the velocity of the fluid decreases, so

$\frac{d w}{d r}=-\left(\frac{S_{t}}{2}\right)^{\frac{1}{n}}\left(\frac{M^{2}}{r}-r\right)^{\frac{1}{n}}$.

Equation (18) is a linear differential equation. Here two cases arise:

Case I: $n=1$ (Newtonian fluid).

Case II: $n \neq 1$ (Power law fluid).

\section{Solution for the Newtonian fluid}

Velocity profile

For $n=1$, the solution of Equation (18) by using boundary condition (16) is,
$w=1-\frac{S_{t}}{4}\left[\left(1-r^{2}\right)+2 M^{2} \ln (r)\right]$,

The vorticity vector $\Omega$ is calculated as:

$\Omega=\nabla \times \mathbf{V}=\left(\frac{S_{t}}{2}\right)\left(\frac{M^{2}}{r}-r\right) \mathbf{j}$,

where $\mathrm{j}$ is the unit vector in $\theta$-direction. We note that the vorticity is zero at the free surface while its magnitude is maximum at the cylinder, given by $\left(\frac{S_{t}}{2}\right)\left(\frac{M^{2}}{r}-r\right)$.

\section{Volume flow rate}

In dimentionless form, the volume flow rate $Q$ is given by,

$Q=\int_{0}^{2 \pi} \int_{1}^{M} r w(r) d r d \theta=2 \pi \int_{1}^{M} r w(r) d r$.

By making use of Equation (19), we acquire,

$Q=\pi\left(M^{2}-1\right)-\frac{S_{t} \pi}{8}\left[4 M^{4} \ln (M)-\left(M^{2}-1\right)^{2}-2 M^{2}\left(M^{2}-1\right)\right]$

\section{Average velocity}

The average film velocity $\bar{V}$ is then given by,

$\bar{V}=\frac{Q}{\pi\left(M^{2}-1\right)}$,

which when use by Equation (22) gives,

$\bar{V}=1-\frac{S_{t}}{8}\left[\frac{4 M^{4} \ln (M)}{\left(M^{2}-1\right)}-3 M^{2}+1\right]$.

Equation (24) gives the net upward flow of liquid. For $\bar{V}>0$,

$1>\frac{S_{t}}{8}\left[\frac{4 M^{4} \ln (M)}{\left(M^{2}-1\right)}-3 M^{2}+1\right]$.

This provides the reasonable estimate for the cylinder velocity to lift a Newtonian fluid.

\section{Solution for the power law fluid}

\section{Velocity profile}

In case of $n \neq 1$ in Equation (18), we have, 
$\frac{d w}{d r}=-\left(\frac{S_{t}}{2}\right)^{\frac{1}{n}}\left(\frac{M^{2}}{r}\right)^{\frac{1}{n}}\left(1-\frac{r^{2}}{M^{2}}\right)^{\frac{1}{n}}, \quad$ where $\left|\frac{r^{2}}{M^{2}}\right|<1$

The expression for velocity field is obtained by solving Equation (26) corresponding to boundary condition (16) as,

$w=1-\left(\frac{S_{t}}{2}\right)^{\frac{1}{n}}\left(\sum_{k=0}^{\infty}\left(\frac{1}{n}\right) \frac{(-1)^{k} M^{-2 k+\frac{2}{n}}}{2 k-\frac{1}{n}+1}\left(r^{2 k-\frac{1}{n}+1}-1\right)\right.$.

By using non-dimensional parameters $S_{r z}=\eta S_{r z}^{*}\left(\frac{U_{0}}{R}\right)^{n}$ and from use of Equation (13) in Equation (3) after dropping '*', we obtain shear stress $S_{r z}$,

$S_{r z}=\left(\frac{d w}{d r}\right)^{n}$

Substituting Equation (17) in foregoing equation, we get,

$S_{r z}=-\left(\frac{S_{t}}{2}\right)\left(\frac{M^{2}}{r}-r\right)$

The $z$-component of the force exerted by the fluid on the cylinder surface is given by

$F_{z}=-\int_{1}^{M}\left(S_{r z}\right)_{r=1} d r$

Inserting the value of $S_{r z}$ from Equation (29) into Equation (30), we get,

$F_{z}=\left(\frac{S_{t}}{2}\right)\left((M-1)^{2}(M+1)\right)$

The vorticity vector $\boldsymbol{\Omega}$ is calculated as:

$\mathbf{\Omega}=\nabla \times \mathbf{V}=\left(\frac{S_{t}}{2}\right)^{\frac{1}{n}}\left(\frac{M^{2}}{r}-r\right)^{\frac{1}{n}} \mathbf{j}$,

where $\mathrm{j}$ is the unit vector in $\theta$-direction. We note that the vorticity is zero at the free surface while its magnitude is maximum at the

cylinder surface given by $\left(\frac{S_{t}}{2}\right)^{\frac{1}{n}}\left(\frac{M^{2}}{r}-r\right)^{\frac{1}{n}}$.

\section{Volume flow rate}

By making use of velocity field given by Equation (27) in (21), we obtain

$$
Q=\pi\left(M^{2}-1\right)-2 \pi\left(\frac{S_{t}}{2}\right)^{\frac{1}{n}} \sum_{k=0}^{\infty}\left(\frac{1}{n} k\right) \frac{(-1)^{k} M^{-2 k+\frac{2}{n}}}{2 k-\frac{1}{n}+1}\left[\frac{\left(M^{2 k-\frac{1}{n}+3}-1\right)}{2 k-\frac{1}{n}+3}-\frac{\left(M^{2}-1\right)}{2}\right] .
$$

\section{Average velocity}

The average film velocity $\bar{V}$ for lift problem investigated comes out to be by using Equation (33) in (23),

$\bar{V}=1-2\left(\frac{S_{t}}{2}\right)^{\frac{1}{n}} \sum_{k=0}^{\infty}\left(\frac{1}{n} k\right) \frac{(-1)^{k} M^{-2 k+\frac{2}{n}}}{2 k-\frac{1}{n}+1}\left[\frac{\left(M^{2 k-\frac{1}{n}+3}-1\right)}{\left(2 k-\frac{1}{n}+3\right)\left(M^{2}-1\right)}-\frac{1}{2}\right]$.

Equation (34) gives the net upward flow of liquid. For $\bar{V}>0$.

$1>2\left(\frac{S_{t}}{2}\right)^{\frac{1}{n}} \sum_{k=0}^{\infty}\left(\frac{1}{n}\right) \frac{(-1)^{k} M^{-2 k+\frac{2}{n}}}{2 k-\frac{1}{n}+1}\left[\frac{\left(M^{2 k-\frac{1}{n}+3}-1\right)}{\left(2 k-\frac{1}{n}+3\right)\left(M^{2}-1\right)}-\frac{1}{2}\right]$.

This equation presents the rational estimate for the cylinder velocity to lift a power law fluid.

\section{Solution for drainage case}

Consider steady, parallel and laminar flow of an incompressible power law fluid down an infinite vertical cylinder. As a result, a thin uniform fluid film of thickness $\delta$ is formed in contact with stationary air. The geomentry of the problem is shown in Figure 2. We choose an $r z$-coordinate system such that $r$-axis is normal to cylinder and $z$-axis along the cylinder axis in the downward direction. We assume that the fluid is non-conducting and completely wets the cylinder. Further, there is no applied (force) pressure driving the flow and fluid falls under the action of gravity, so the governing Equation (14) becomes,

$\frac{1}{r} \frac{d}{d r}\left(r\left(\frac{d w}{d r}\right)^{n}\right)=-S_{t}$,

along with the boundary conditions,

$\frac{d w}{d r}=0 \quad$ at $\quad r=M$

$w=0 \quad$ at $\quad r=1$.

Integrating Equation (36) with respect to $r$ and using boundary conditions (37), we obtain, 


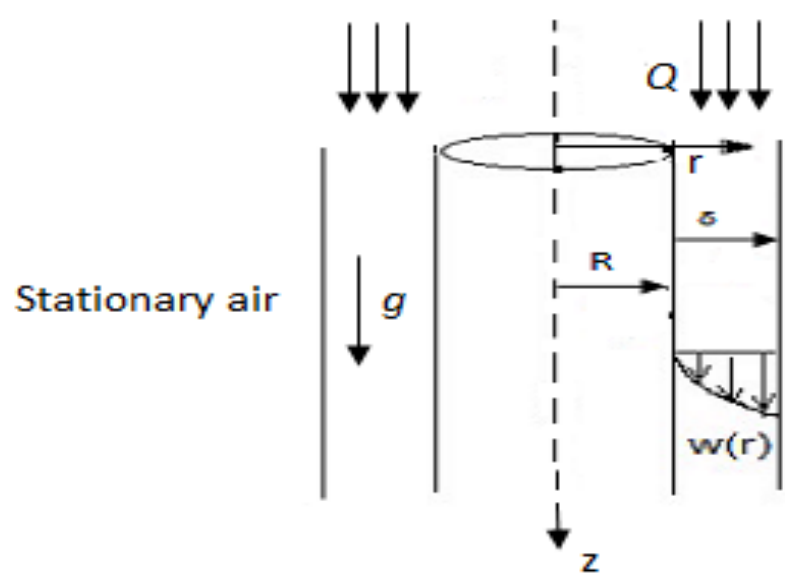

Figure 2. Geometry of the thin film flow down a vertical cylinder.

$\frac{d w}{d r}=\left(\frac{S_{t}}{2}\right)^{\frac{1}{n}}\left(\frac{M^{2}}{r}-r\right)^{\frac{1}{n}}$

which is a linear differential equation. Here two cases arise:

Case-I: $n=1$, we recoverd the result of Newtonian fluid given in Papanastasiou et al. (2000).

Case-II: $n \neq 1$, power law fluid.

\section{Solution for the power law fluid}

\section{Velocity profile}

For $n \neq 1$, from Equation (39), we have,

$$
\frac{d w}{d r}=\left(\frac{S_{t}}{2}\right)^{\frac{1}{n}}\left(\frac{M^{2}}{r}\right)^{\frac{1}{n}}\left(1-\frac{r^{2}}{M}\right)^{\frac{1}{n}}, \quad \text { where }\left|\frac{r^{2}}{M^{2}}\right|<1
$$

The solution of Equation (40) by using binomial series and applying boundary condition (38) is,

$$
w=\left(\frac{S_{t}}{2}\right)^{\frac{1}{n}}\left(\sum_{k=0}^{\infty}\left(\frac{1}{n}\right) \frac{(-1)^{k} M^{-2 k+\frac{2}{n}}}{2 k-\frac{1}{n}+1}\left(r^{2 k-\frac{1}{n}+1}-1\right)\right) .
$$

Substitute Equation (40) in Equation (28), we get,

$$
S_{r z}=\left(\frac{S_{t}}{2}\right)\left(\frac{M^{2}}{r}-r\right)
$$

The $z$ - component of the force exerted by the fluid on the cylinder will be obtained after inserting the value of $S_{r z}$ from Equation (42) into Equation (30), we will get,

$$
F_{z}=-\left(\frac{S_{t}}{2}\right)\left((M-1)^{2}(M+1)\right)
$$

The vorticity vector $\boldsymbol{\Omega}$ is calculated as:

$\mathbf{\Omega}=\nabla \times \mathbf{V}=-\left(\frac{S_{t}}{2}\right)^{\frac{1}{n}}\left(\frac{M^{2}}{r}-r\right)^{\frac{1}{n}} \mathbf{j}$,

where $\mathrm{j}$ is the unit vector in $\theta$-direction. We note that the vorticity is zero at the free surface while its magnitude is maximum at the

cylinder given by $\left(\frac{S_{t}}{2}\right)^{\frac{1}{n}}\left(\frac{M^{2}}{r}-r\right)^{\frac{1}{n}}$.

\section{Volume flow rate}

The volume flow rate $Q$ for drainage problem can be obtained by using Equation (41) in (21),

$$
Q=2 \pi\left(\frac{S_{t}}{2}\right)^{\frac{1}{n}} \sum_{k=0}^{\infty}\left(\frac{1}{n}\right) \frac{(-1)^{k} M^{-2 k+\frac{2}{n}}}{2 k-\frac{1}{n}+1}\left[\frac{\left(M^{2 k-\frac{1}{n}+3}-1\right)}{2 k-\frac{1}{n}+3}-\frac{1}{2}\left(M^{2}-1\right)\right] .
$$

\section{Average velocity}

The average film velocity $\bar{V}$ for drainage problem can be calculated by using Equation (45) in (23),

$$
\bar{V}=2\left(\frac{S_{t}}{2}\right)^{\frac{1}{n}} \sum_{k=0}^{\infty}\left(\frac{1}{n} k\right) \frac{(-1)^{k} M^{-2 k+\frac{2}{n}}}{2 k-\frac{1}{n}+1}\left[\frac{\left(M^{2 k-\frac{1}{n}+3}-1\right)}{\left(2 k-\frac{1}{n}+3\right)\left(M^{2}-1\right)}-\frac{1}{2}\right] .
$$

\section{RESULTS AND DISCUSSION}

The quantitative effects of power law index $n$, parameter $M$ and Stokes' number $S_{t}$ on velocity profile, shear stress and flow rate are presented graphically in Figures 3 to 19. The variation of axial velocity for $n, M$ and $S_{t}$ for both Newtonian and power law fluid in case of lift is displayed in Figures 3 to 8 . In Figures 3 to 8 , we observed that with an increase in $n, M$ and $S_{t}$, velocity profile decreases. The difference of $n, M$ and $S_{t}$ for drainage of fluid film in Figures 9 to 11 have been plotted, in which it is observed that the velocity of fluid film increases significantly for a change in parameters of 


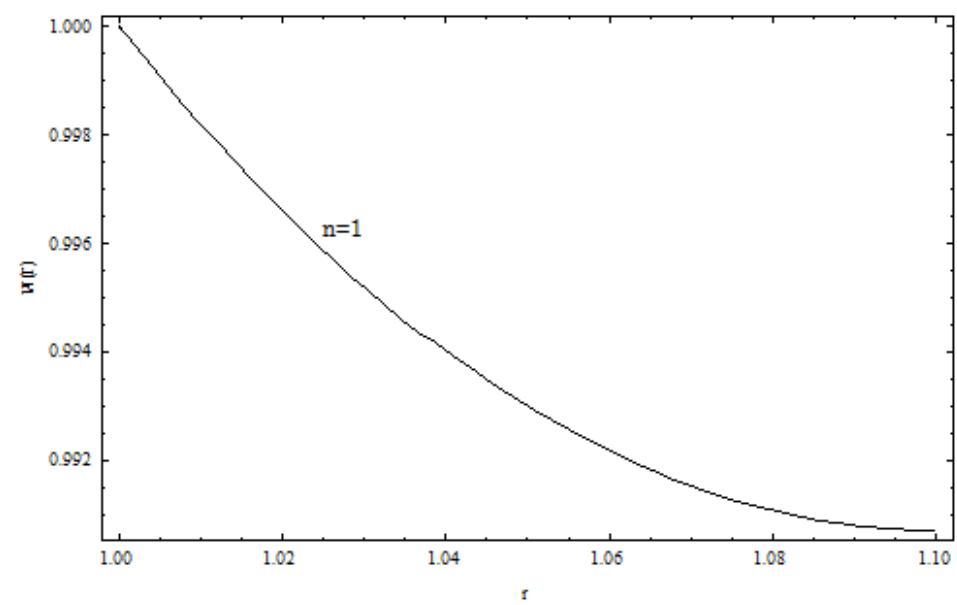

Figure 3. Velocity profile for Newtonian fluid for lift in thin film flow when $S_{t}=0.8, M=1.1$.

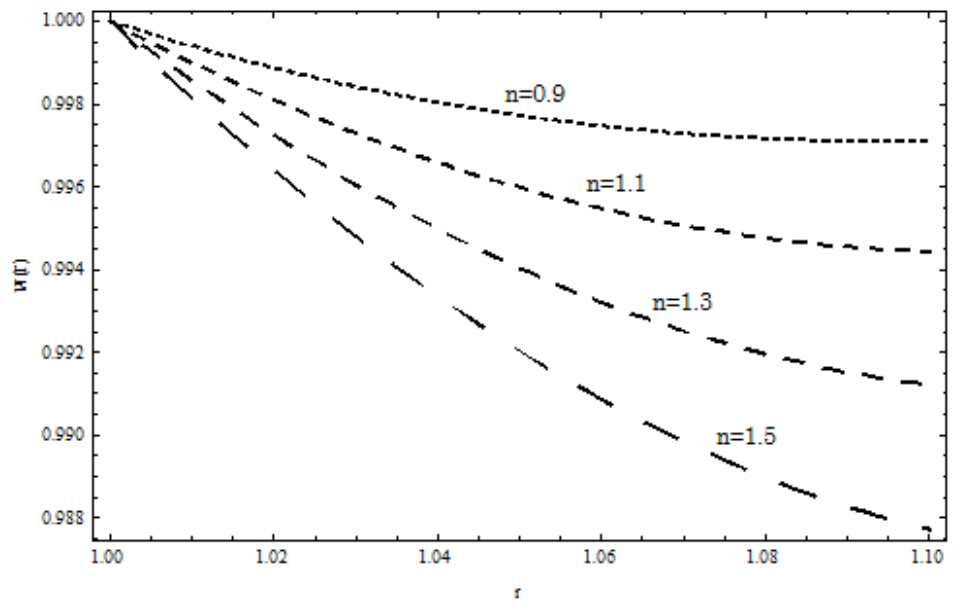

Figure 4. Velocity profile for power law fluid for lift in thin film flow for different values of $n$, when $S_{t}=0.8, M=1.1$.

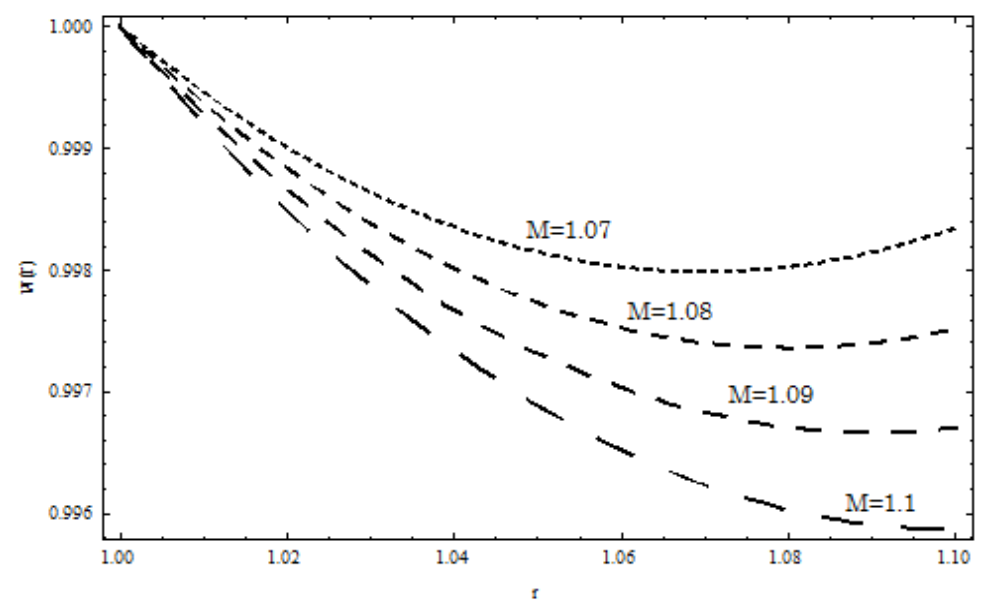

Figure 5. Velocity profile for Newtonian fluid for lift in thin film flow for different values of $M$, when $S_{t}=0.8$. 


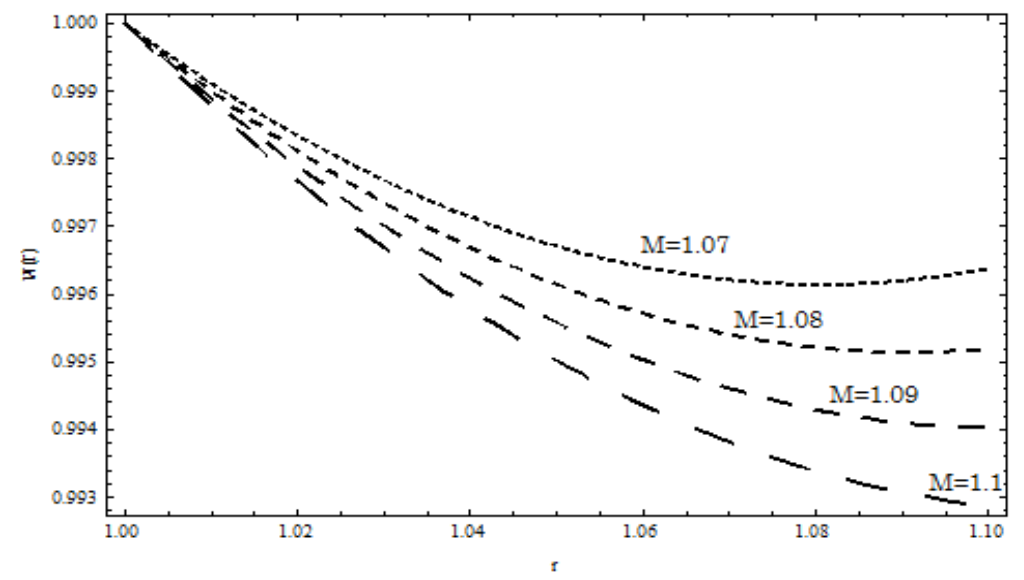

Figure 6. Velocity profile for power law fluid for lift in thin film flow for different values of $M$, when $S_{t}=0.8, n=1.2$.

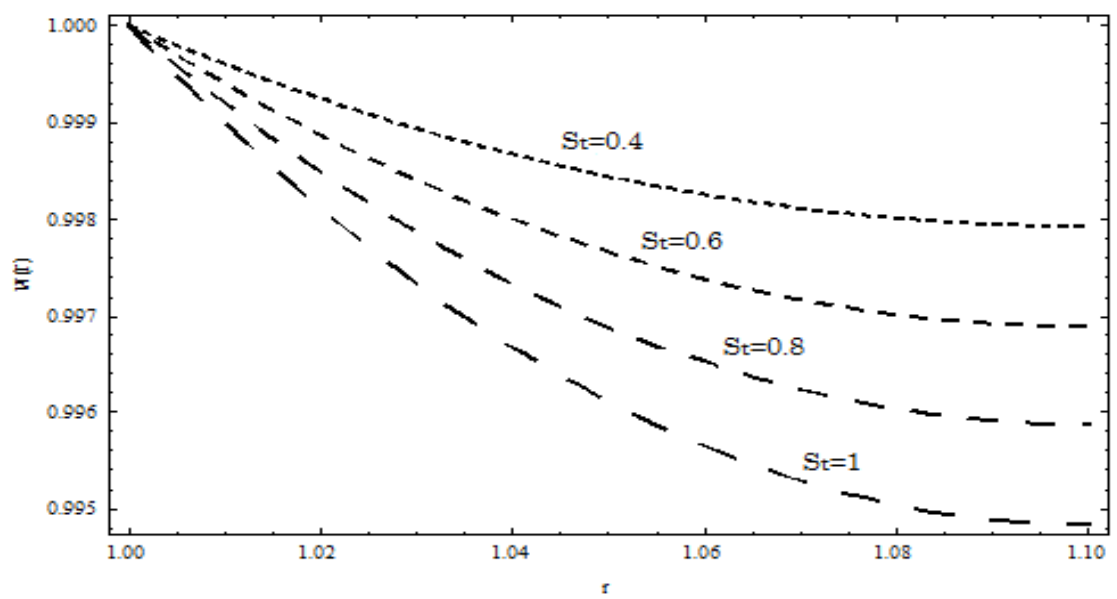

Figure 7. Velocity profile for Newtonian fluid for lift in thin film flow for different values of Stokes number $S_{t}$, when $\mathrm{M}=1.1$.

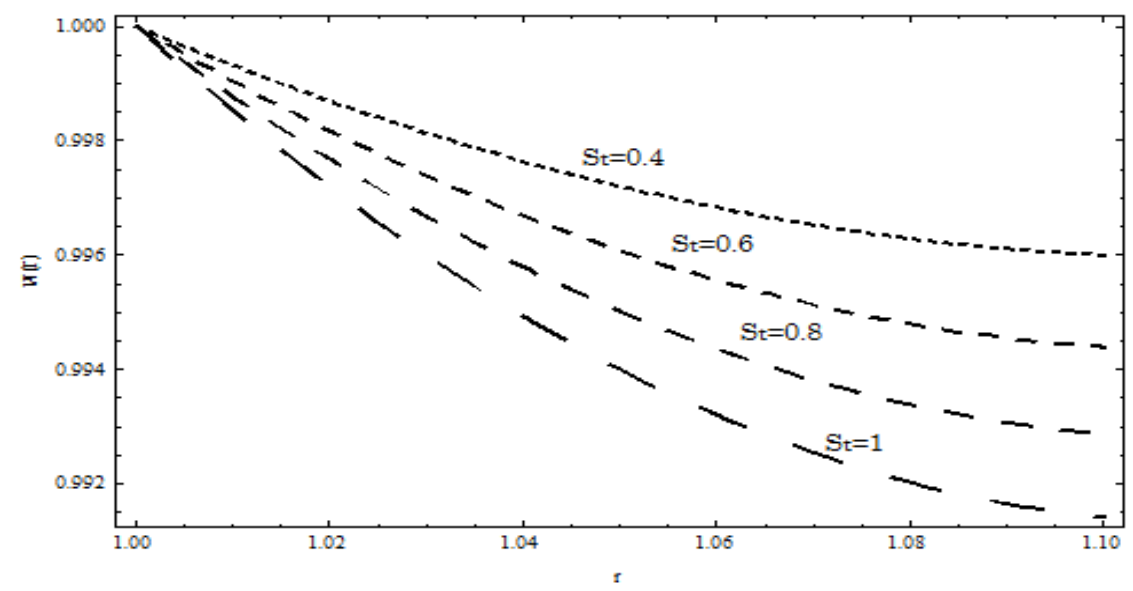

Figure 8. Velocity profile for power law fluid for lift in thin film flow for different values of Stokes number $S_{t}$, when $M=1.1, n=1.2$. 


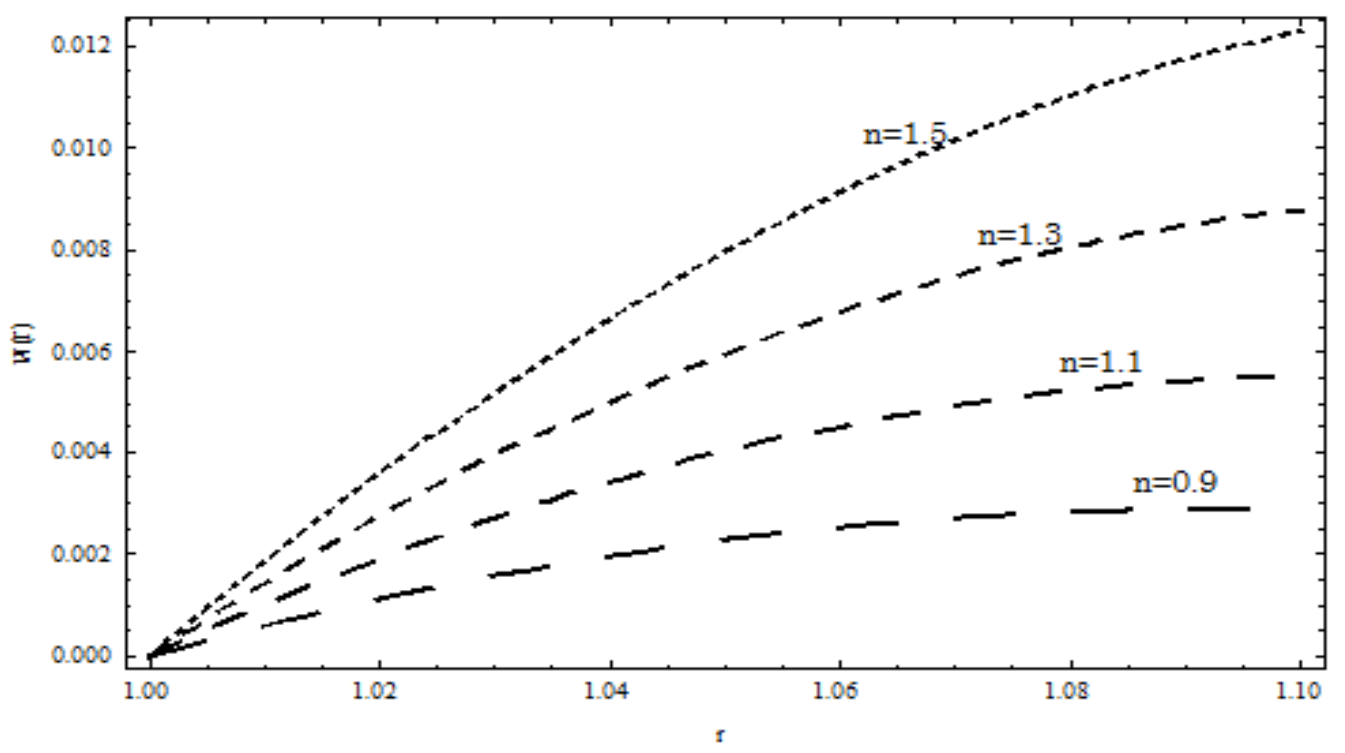

Figure 9. Velocity profile for power law fluid for drainage in thin film flow for different values of $n$, when $S_{t}=0.8, M=1.1$.

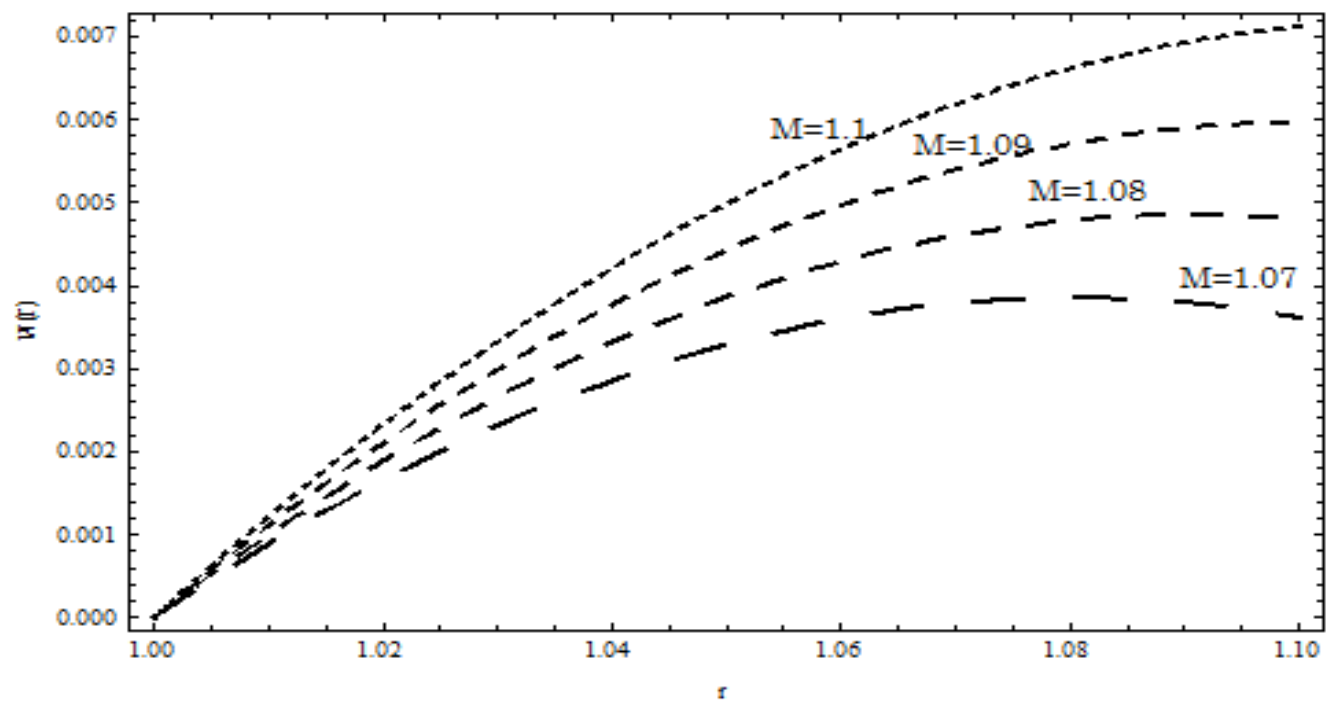

Figure 10. Velocity profile for power law fluid for drainage in thin film flow for different values of $M$, when $S_{t}=0.8, n=1.2$.

power law fluid model. The variation of shear stress in case of lift and drainage at different dissimilarity parameters $M$ and $S_{t}$ is demostrated in figures 12 to 15. Here it is observed that both dissimilarity parameters are directly proportional to the shear stress for drainage case and inverselly proportional for lift case. Difference for $n, M$ and $S_{t}$ for flow rate is also observed for drainage case in Figures 16 to 19, in which we observed that, flow rate is also directly proportionsl for all parameters for drainage case and inversely proportional for lift case.

\section{Conclusion}

We have presented results for the thin film flow field of a fluid, which is called the power law fluid, on a vertical cylinder for lift and drainage problem. The resulting nonlinear differential equation has been solved by 


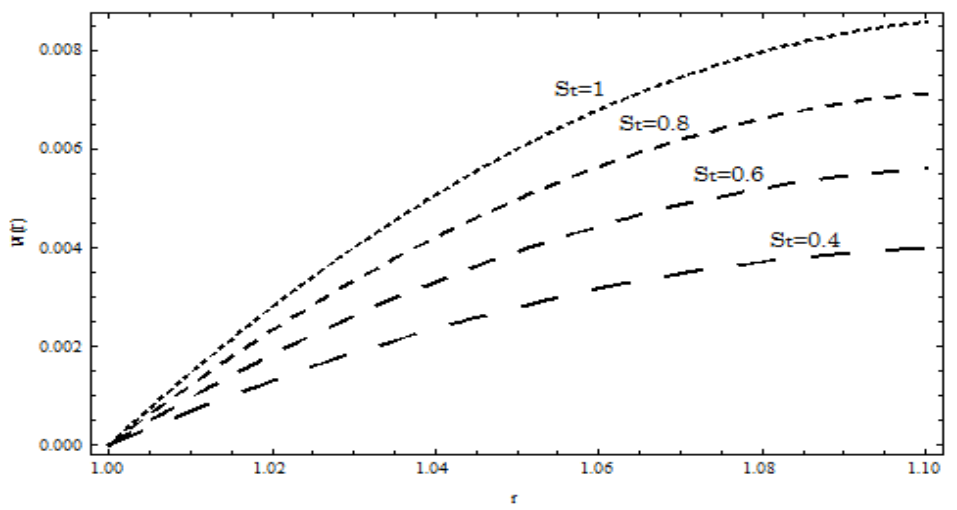

Figure 11. Velocity profile for power law fluid for drainage in thin film flow for different values of $S_{t}$, when $M=1.1, n=1.2$.

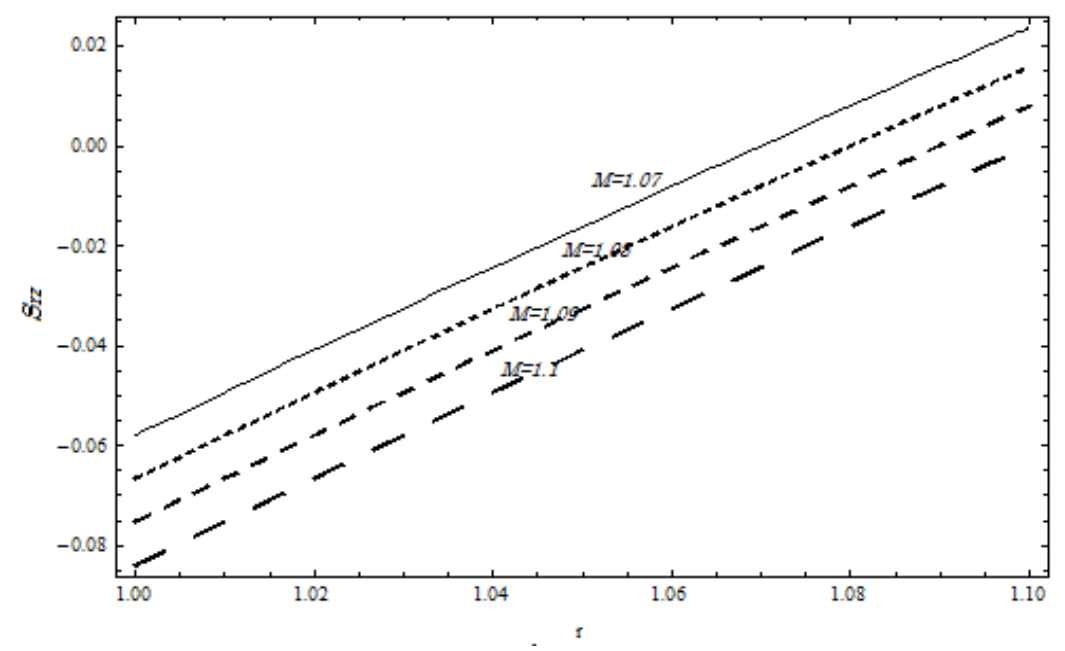

Figure 12. The effect of $M$ on shear stress for lift, when $S_{t}=0.8$.

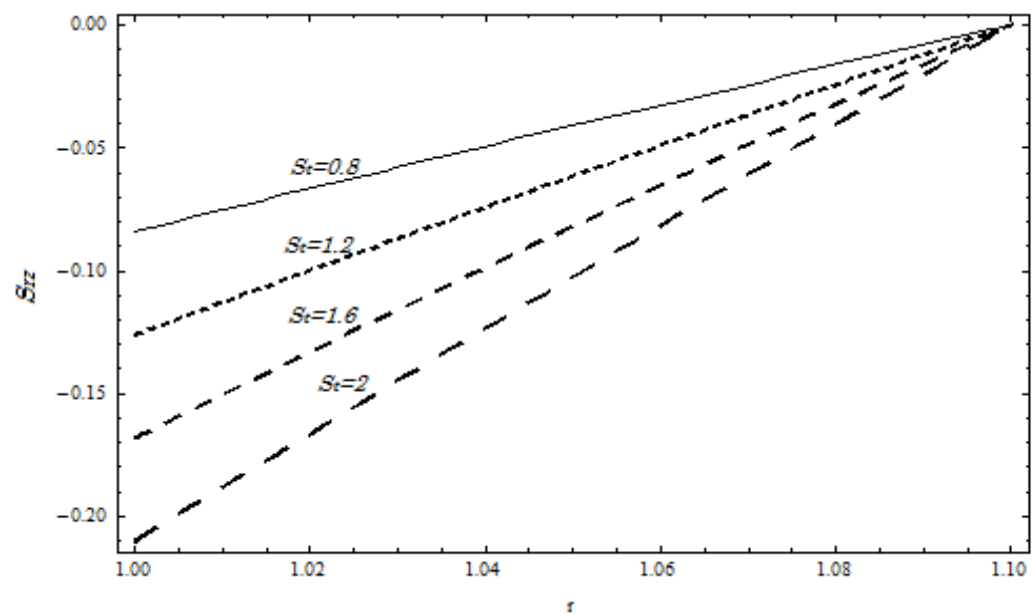

Figure 13. The effect of $S_{t}$ on shear stress for lift, when $M=1.1$. 


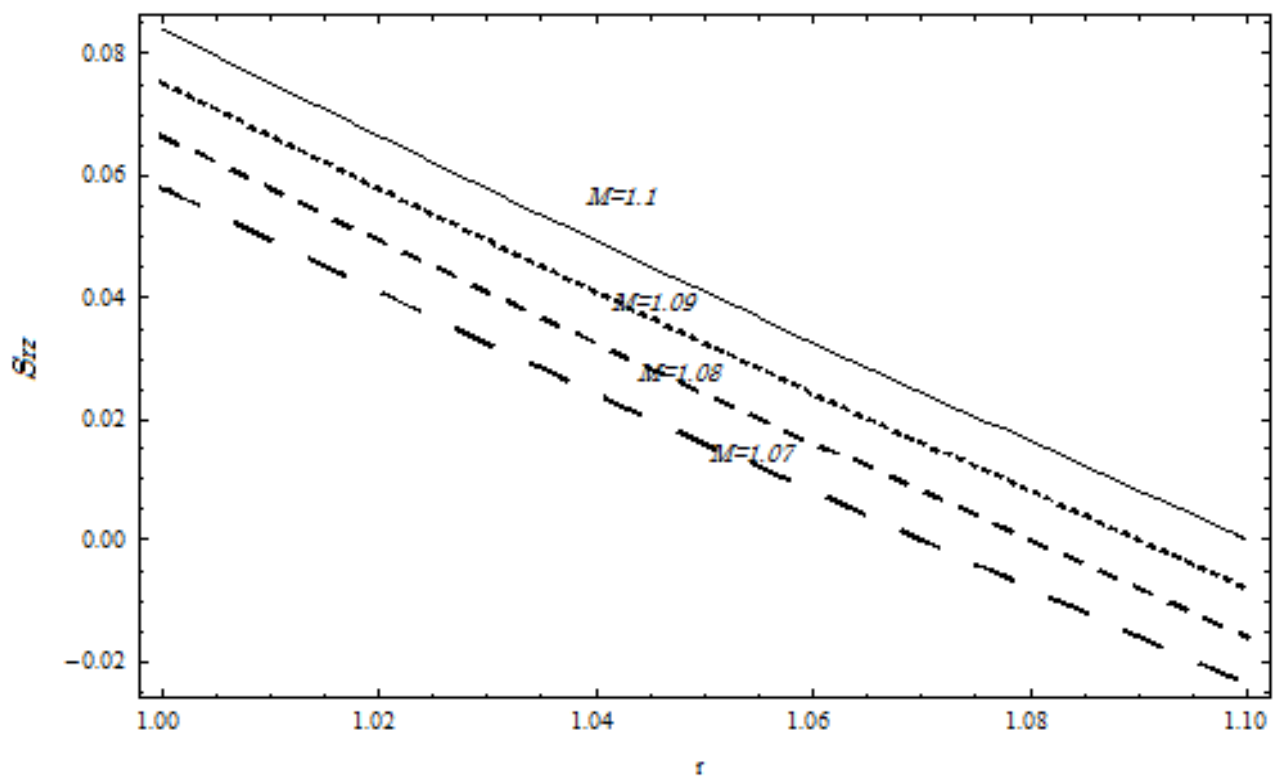

Figure 14. The effect of $M$ on shear stress for drainage, when $S_{t}=0.8$.

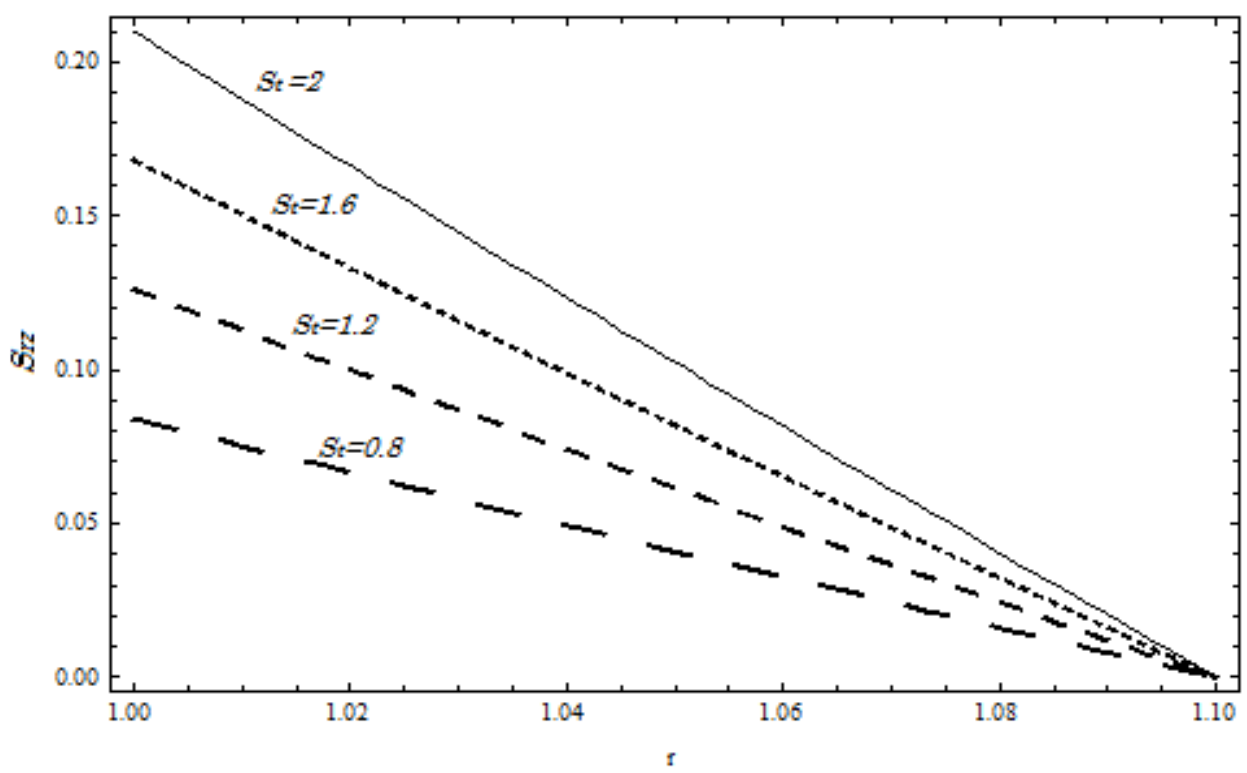

Figure 15. The effect of $S_{t}$ on shear stress for drainage, when $M=1.1$.

binomial series method, which is reliable method for the proposed problem. The velocity profile, volume flow rate and average velocity have been derived analytically.

NOMENCLATURE: $\mathbf{A}_{1}$, Rivilin-Ericksen tensor; $\frac{D}{D t}$, material time derivative; f, body force; $F_{z}$, Force exerted by the fluid; $\mathbf{g}$, Acceleration due to gravity; $\delta$, thickness of fluid film; $\boldsymbol{n}$, Power law index; $\boldsymbol{P}$, dynamic pressure; $Q$, volume flow rate; $\mathbf{S}$, extra stress tensor; $\boldsymbol{S}_{t}$, Stokes' number; $\boldsymbol{t}$, time. $\bar{V}$, average velocity; $\mathbf{V}$, velocity vector. $\boldsymbol{w}$, Axial component of the velocity field $\mathbf{v} . \eta$, dynamic viscosity; $\mu_{\text {eff }}$, power law fluid parameter; $\rho$, density of the fluid; $\boldsymbol{\Omega}$, vorticity vector. 


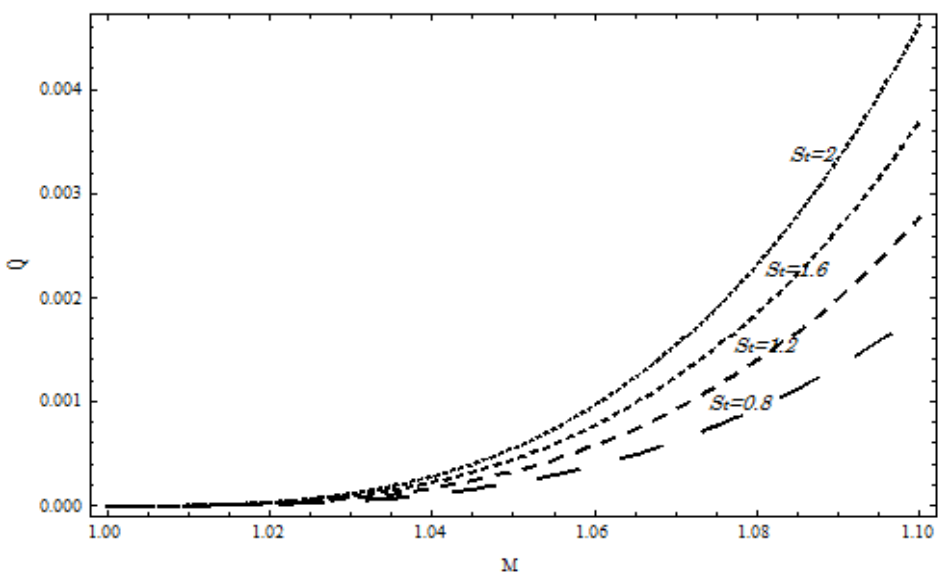

Figure 16. The effect of $S_{t}$ on flow rate for Newtonian fluid for drainage.

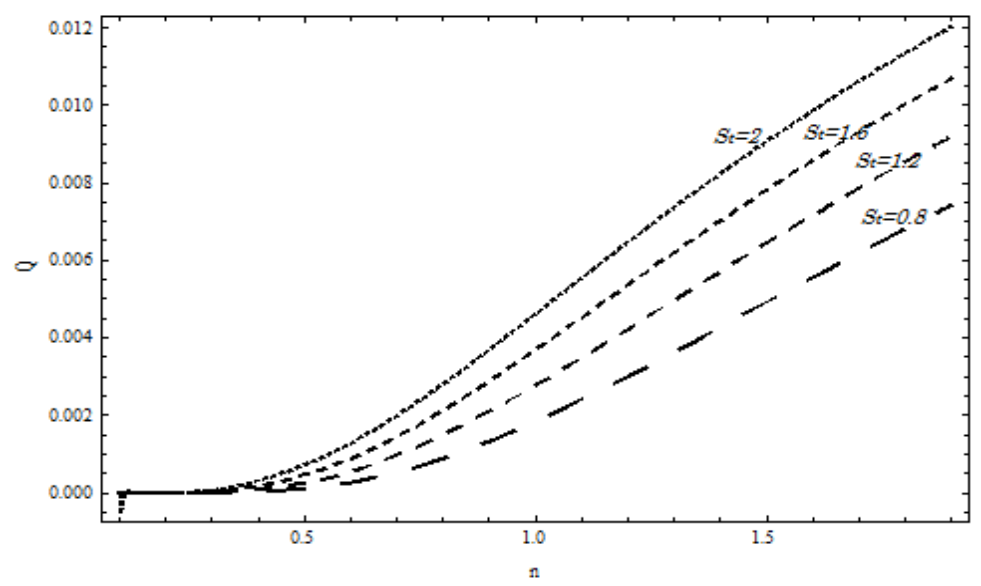

Figure 17. The effect of $S_{t}$ on flow rate for power law fluid for drainage, when $M=1.1$.

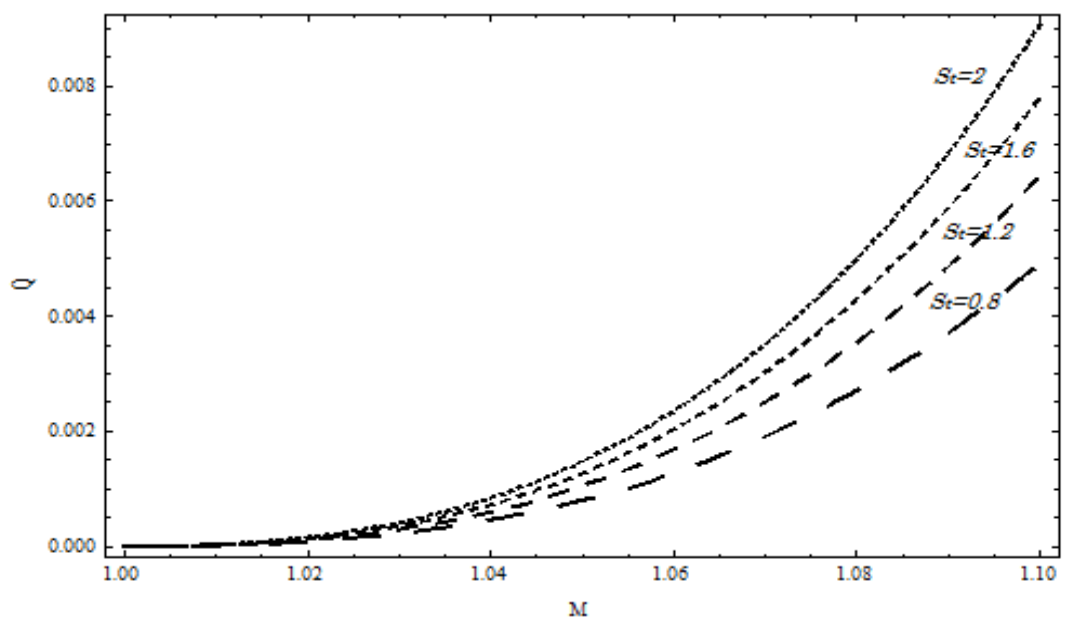

Figure 18. The effect of $S_{t}$ on flow rate for power law fluid for drainage, when $n=1.1$. 


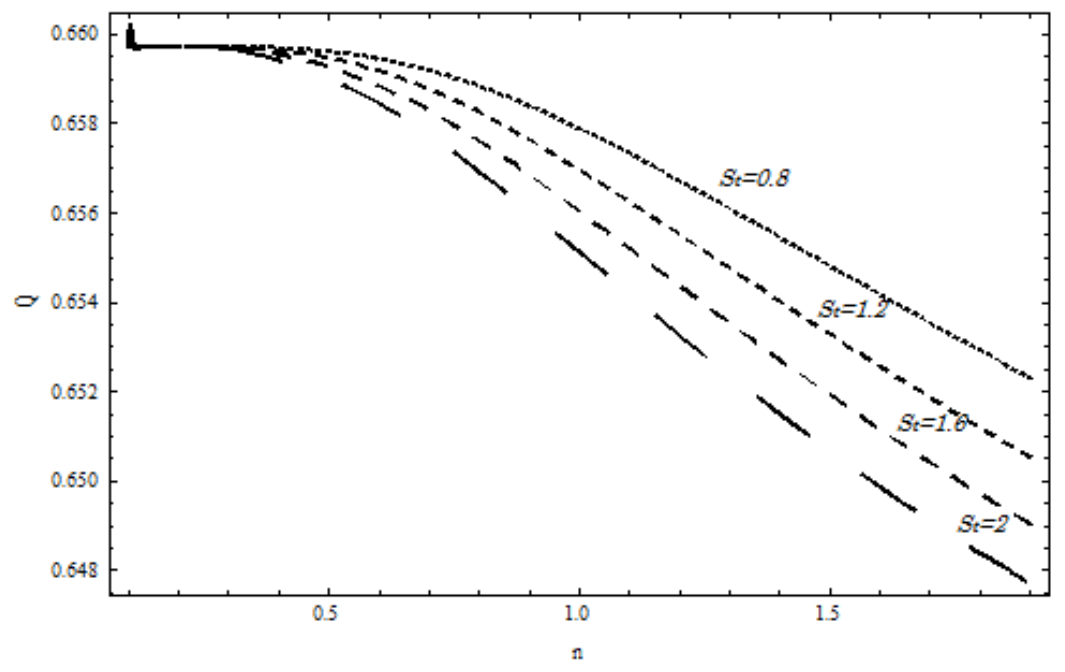

Figure 19. The effect of $S_{t}$ on flow rate for power law fluid for lift, when $M=1.1$.

\section{REFERENCES}

Bird RB, Armstrong RC, Hassager O (1987). Dynamics of polymeric liquids, Fluid Mechnics, John Wiley and Sons, 1: 173.

Coolidge JL (1949). The story of the Binomial Theorem. Harvard University (March).

Deshpande NS, Barigou M (2001). Vibrational flow of non-Newtonian fluids. Chem. Eng. Sci. 56:3845-3853.

Dita P, Grama N (1997). On Adomian's decomposition method for solving differential equations. arXiv:solv-int/9705008 V. 1:14.

Ghoreishy MHR, Razavi NM (1998). A Three-dimensional Finite Element Analysis of a Power-law Fluid in an Extrusion Die, Iran. Polym. J. 7(4):1026-1263/98.

Hayat T, Sajid M (2007). On analytic solution for thin film flow of a fourth grade fluid down a vertical cylinder. Phys. Lett. A 361:316-322.

Hayat T, Sajid M (2008). Thin film flow of an Oldroyd 8-constant fluid: An exact solution. Phys. Lett. A. 372:1827-1830.

$\mathrm{He} \mathrm{JH}$ (2008). An elementary introduction to recently developed asymptotic methods and nanomechanics in textile engineering. Int. J. Mod. Phys. B. 22:3487.

$\mathrm{He} \mathrm{JH}$ (2011). A short remark on fractional variation iteration method, Phys. Lett. A. 375(38):3362-3364

$\mathrm{He} \mathrm{JH}$ (2012). Homotopy perturbation method with an auxiliary term, Abster. Appl. Anal. doi:10.1155/2012/857612. p. 7.

Jie C, Xi-Yun L (2006). Numerical investigation of the non-Newtonian pulsatile blood flow in a bifurcation model with a non-planar branch, J. Biomech. 39:818-832.

Kapur JN (1963). Flows of Power-Law Fluids Past a Flat Plate with Uniform Suction and Between Two Parallel Plates with Uniform Suction and Injection. J. Phys. Soc. Japan. 18(4):578-581.

Kemiha M, Frank X, Poncin S, Li HZ (2006). Origin of the negative wake behind a bubble rising in non-Newtonian fluids. Chem. Eng. Sci. 61:4041-4047.

Mahomed FM, Hayat T, Momoniat E, Asghar S (2007). Gliding motion of bacterium in a non-Newtonian slime. Nonlinear Analysis: Real World Applications. pp. 853-864.

Nejat A, Jalali A, Sharbatdar M (2011). A Newton-Krylov finite volume algorithm for the power-law non-Newtonian fluid flow using pseudocompressibility technique. J. Nonnewton. Fluid. Mech. 166:11581172.
Papanastasiou TC, Georgin GC, Andreas NA (2000). CRC Press Boca Raton London Newyork Washington, D.C. 6: 23.

Siddiqui AM, Ahmad M, Ghori QK (2007). Thin film flow of nonNewtonian fluids on a moving belt, Chaos. Solutions and Fractals 33: 1006-1016.

Siddiqui AM, Mahmood R, Ghori QK (2006a). Some exact solutions for the thin film flow of a PTT fluid, Phys. Lett. A. 356:353-356.

Siddiqui AM, Mahmood R, Ghori QK (2006b). Homotopy perturbation method for thin film flow of a fourth grade fluid down a vertical cylinder, Phys. Lett. A. 352:404-410.

Yong-Li C, Xu-Dong C, Ke-Qin Z (2009). A gray lattice Boltzmann model for power-law fluid and its application in the study of slip velocity at porous interface. J. Nonnewton. Fluid Mech. 159:130-136. 